\section{E0048 CYP2E1 INCREASES OXIDATIVE STRESS AND INDUCES APOPTOSIS OF CARDIO MYOCYTES IN TRANSGENIC MICE}

doi:10.1136/hrt.2010.208967.48

\begin{abstract}
${ }^{1}$ Zhang Wei, ${ }^{1}$ Lu Dan, ${ }^{1}$ Dong Wei, ${ }^{1}$ Zhang Li, ${ }^{1}$ Zhang Xiaojuan, ${ }^{1}$ Quan Xiongzhi, ${ }^{2}$ Xu Yanfeng, ${ }^{2}$ Ma Chunmei, 'Lian Hong, 'Zhang Lianfeng. ${ }^{1}$ Key Laboratory of Human Disease Comparative Medicine Ministry of Healthinstitute of Laboratory Animal Science Chinese Academy of Medical Sciences \& Comparative Medical Center Peking Union Medical College; ${ }^{2}$ Key Laboratory of Human Diseases Animal Model State Administration of Traditional Chinese Medicine Institute of Laboratory Animal Science Chinese Academy of Medical Sciences \& Comparative Medical Center Peking Union Medical College
\end{abstract}

Objective Cytochrome P450 2E1 (CYP2E1) is an effective generator of reactive oxygen species, such as the superoxide anion radical and hydrogen peroxide. The expression of CYP2E1 varies with the progression of myocardial ischaemia and cardiomyopathy. This paper examined the heart specific CYP2E1 transgenic mice to study the effect of CYP2E1 on DCM.

Methods The expression of CYP2E1 in both hypertrophic cardiomyopathy (HCM) and DCM mice were analysed using RT-PCR and western blot on cTnTR92O and cTnTR141W transgenic mice. The cDNA of CYP2E1 was amplified by RT-PCR from the mice heart, and del inserted into the downstream of $\alpha$-MHC promoter to construct the CYP2E1 expression vector. The transgenic mice were created by the method of microinjection. And they were del crossed with cTnTR141W transgenic mice. The cardiac structure and function were analysed with $\mathrm{M}$-mode echocardiography. Survival data of the experimental mice were recorded. Pathologic changes were observed by light microscopy. The contents of hydrogen peroxide (H2O2), malonaldehyde malondialdehyde (MDA), reduced glutathione (GSH) as well as the total anti oxidation capable (T-AOC) were detected by spectrophotometry. Myocyte apoptosis was analysed by in situ terminal dUTP nick end-labelling (TUNEL) stain. Results In the current paper, it was indicated that expression of CYP2E1 was lightly up-regulated in HCM hearts from cTnTR92O transgenic mice and was strongly down-regulated in DCM hearts from cTnTR141W transgenic mice at 3 months old. The transgenic expression of CYP2E1 reduced mortality of CYP2E1 transgenic mice by $7.5 \%$ and increased the mortality of CTnTR $141 \mathrm{~W}$ transgenic mice from $10 \%$ to $45 \%(n=40, p<0.01)$. The expression of CYP2E1 also brought about increases in left ventricular volumes, diameters, and decreases in left ventricular walls, ejection fraction and fractional shortening, as well as increases in myocyte disarray and interstitial fibrosis for both of CYP2E1 and CYP2E1XcTnTR141W transgenic mice. The levels of $\mathrm{H} 2 \mathrm{O} 2$ and MDA were increased and the levels of GSH and T-AOC were strongly reduced in both of CYP2E1 and CYP2E1 $\times c$ TnTR141W transgenic mice. In addition, myocyte apoptosis del increased 7-fold in the CYP2E1 transgenic mice compared with WT mice $(n=3, p<0.01)$ and increased 1.7-fold in the CYP2E1 $\times$ cTnTR141W transgenic mice compared with cTnTR141W transgenic mice $(n=3, p<0.01)$

Conclusions Expression of CYP2E1 was regulated in the progression of transgenic cardiomyopathy mice. CYP2E1-mediated oxidative stress and myocyte apoptosis may play an important role in aggravating the DCM phenotype.

\section{e0049 E2F1 STABILISES P53 TO SUPPRESS VEGF EXPRESSION AND NEOVASCULARIZATION IN THE ISCHAEMIC MYOCARDIUM}

doi:10.1136/hrt.2010.208967.49

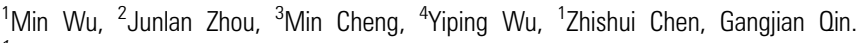
${ }^{1}$ Feinberg Cardiovascular Research Institute Northwestern University Feinberg School of Medicine 2.1. Feinberg Cardiovascular Research Institute Northwestern University
Feinberg School of Medicine; ${ }^{2}$ Department of Cardiology, Union Hospital Tongji Medical College Huazhong University of Science And Technology; ${ }^{3}$ Department of Plastic Surgery Tongji Hospital Tongji Medical College Huazhong University of Science And Technology; ${ }^{4}$ Institute of Organ Transplantation Tongji Hospital Tongji Medical College Huazhong University of Science And Technology

Objective We have previously shown that genetic deletion of the transcription factor E2F1 increases the expression of vascular endothelial growth factor (VEGF) and enhances blood flow recovery in the ischaemic limb (Oin et al, PNAS 2006). However, the physiological significance of this regulation in ischaemic heart disease and the molecular mechanisms of E2F1-mediated VEGF regulation are still unknown. The purpose of this study is to understand the role of E2F1 in cardiac neovascularization following ischaemic injury

Methods and results Myocardial infarction (MI) was induced by surgical ligation of the Left Anterior Descending (LAD) coronary artery in wild-type (WT) and E2F1-/- mice. At day 5 after surgery, angiogenic factors at the infarct border zone were analysed by qRTPCR and Western blotting. At day 28, the vascular density and infarct size were evaluated histologically. VEGF $\mathrm{mRNA}$ and protein levels were significantly higher in E2F1-/- than in WT mice $(\mathrm{p}<0.01, \mathrm{n}=5)$. E2F1 $-/-$ mice displayed a greater vessel density in the infarct border area $(\mathrm{p}<0.01, \mathrm{n}=5)$ and a smaller infarct size $(\mathrm{p}<0.01, \mathrm{n}=15)$. In vitro, hypoxia treatment $(0.5 \% \mathrm{O} 2$ for $24 \mathrm{~h})$ increased VEGF mRNA expression to a higher level in E2F1-/cardiac fibroblasts than in WT control cells $(p<0.01, n=3)$. Overexpression of E2F1 suppressed the hypoxia-induced VEGF promoter activity in WT cells, however, (del) but not in p53-/- cells, suggesting that p53 is required for E2F1 to suppress VEGF transcription. Hypoxia treatment $(0.5 \% \mathrm{O} 2)$ for $24 \mathrm{~h}$ dramatically increased the level of both E2F1 and p53 proteins; overexpression of E2F1 further enhanced the hypoxia-induced accumulation of p53. To understand whether E2F1 regulates p53 protein stability, we treated WT and E2F1-/- cardiac fibroblasts with hypoxia for $6 \mathrm{~h}$, pulsed the cells with cyclohexamide $(40 \mathrm{mg} / \mathrm{ml})$ and chased p53 degradation. The p53 protein level declined gradually in WT cells (half-life: $\sim 4 \mathrm{~h}$ ), but, significantly faster in E2F1-/- cells (half-life: $\sim 1 \mathrm{~h})(\mathrm{p}<0.01$ at 1,2 , and $4 \mathrm{~h}, \mathrm{n}=4$ ). Interestingly, addition of Lactacystin significantly delayed the rates of p53 degradation in both WT and E2F1-/ - cells and eliminated the difference between the two groups of cells, suggesting that under hypoxia, E2F1 promotes p53 accumulation by attenuating its ubiquitin-proteasomal degradation. Furthermore, co-immunoprecipitation (co-IP) experiments indicated that hypoxia treatment induced physical associations between E2F1 and p53. In the E2F1-/- fibroblasts transiently transfected with HA-tagged E2F1 or E2F1 truncation mutants, p53 co-precipitated with the ectopically expressed WT E2F1 and the E2F1 mutants with deletions in the transactivaton domain and/or DP-dimerisation domain, but not with the E2F1 mutant with a deletion in the $\mathrm{N}$-terminus (amino acids $1-109$ ), suggesting that the $\mathrm{N}$-terminal region is essential for E2F1 to interact with p53.

Conclusions The E2F1 stabilises p53 protein, thereby suppressing VEGF expression and new vessel formation in the ischaemic heart. Targeting E2F1:p53 interaction (eg, by E2F1 N-terminal peptide) may protect heart from ischaemic injury.

*Note: Results and Methods were mixed.

\section{e0050 EFFECT OF BOSENTAN ON CAROTID ARTERY RESTENOSIS IN RAT AND CORRELATION OF SERUM VEGF}

doi:10.1136/hrt.2010.208967.50

Yanhong Zhang, Guiping Wu, Xin Fu, Ying Li, Xiaoting Ou. Department of Cardiology, Shenzhou Hospital, Shenyang Medical College, Shenyang, China

Objective To investigate the effect of Bosentan on carotid artery restenosis in rat and correlation of serum vascular endothelia growth factor (VEGF). 
Methods 36 Wistar rats were divided randomly into three groups: control group ( $n=12)$, angioplasty group $(n=12)$ and Bosentan (BA) with angioplasty group $(n=12)$. Bosentan was administrated del to rats of BA with angioplasty group. The balloon catheter injury was performed on left common carotid artery of rat by imitating the process of angioplasty. After 7 days and 14 days, neointimal area (NIA) and stenosis index (SI) were calculated. Prior to angioplasty and 7 days after balloon injury, serum VEGF were assessed. In another experiment, 65 Wistar rats were divided randomly into three groups: control group, angioplasty group and Bosentan with angioplasty group. Bosentan was administrated to rats of Bosentan (BA) with angioplasty group. The balloon catheter injury was performed on left common carotid artery of rat by imitating the process of angioplasty. The process of neointimal and media hyperplasia was observed and $\alpha$-action and PCNA expressions were determined.

Results NIA and SI of BA with angioplasty were significantly decreased compared with angioplasty group $(p<0.001)$. Serum VEGF level significantly increased 7 days after balloon injury both in angioplasty group and BA group. The increase range in BA with angioplasty group was higher than that of the angioplasty group $(p<0.01)$. The serum VEGF level 7 days after injury had linearly negative correlation with SI. In the second experiment, arterial neointima hyperplasia reached delsummit at 28 days in the angioplasty group and 14 days in the BA with angioplasty group. Neointimal and media area of BA with angioplasty at different times (14th, 28th, 45thday) were significantly decreased compared with angioplasty group $(p<0.001)$. The rate of PCNA positive cell increased statistically in BA with angioplasty group than in angioplasty group at 14 th day $(p<0.01)$. The rat of $\alpha$-actin positive cell increased significantly in BA with angioplasty group compared with angioplasty group $(\mathrm{p}<0.01)$ at 14 th day.

Conclusions Bosentan may be effective on artery restenosis by inhibiting neointimal hyperplasia, increasing the serum VEGF level, reducing the proliferation, migration and transconformation of vascular smooth muscle cells.

Note: Combination of paper e0367 and e0371.

\section{e0051 MIR214 IS UPREGULATED DURING VENTRICULAR REMODELLING POST MI}

doi:10.1136/hrt.2010.208967.51

Bing Shi, Lin Mi, Haiyi Yu, Wei Gao. Peking University Third Hospital

Objective MicroRNAs (miRNAs) are endogeneous, single-stranded non-coding RNA molecules about 22 nucleotides long, del regulating target gene expression post-transcriptionally by base pairing with specific binding sites located in the 3' - untranslated regions (UTRs) of downstream target mRNAs. miRNAs play important roles in the regulation of a multitude of physiological functions such as cell differentiation, proliferation, apoptosis and immune response. Recent studies suggest that aberrant expression of miRNAs is associated with cardiovascular diseases. Those miRNAs exhibit unique spatial expression patterns that might become biomarker of diagnosis and target of treatment of ventricular remodelling. In present study, the expression level of miR-214 during ventricular remodelling post MI was detected.

Methods Rats underwent left descending coronary ligation or sham surgery. Rats with MI was assigned to two groups $(n=5)$. Realtime PCR was developed to detect the expression of miR-214 in myocardium and plasma.

Results The expression level of miR-214 in both myocardium and plasma were up-regulated in the 14 th and 28 th day post MI. Compared to sham group, the expression level of miR-214 in myocardium increased by $33 \%(1.33 \pm 0.12$ vs $1.00 \pm 0.02, p<0.01)$ in the 14 th day and by $88 \%(1.88 \pm 0.08$ vs $1.00 \pm 0.02, p<0.01)$ in the 28 th day post MI. Compared to sham group, the expression of miR214 in plasma increased by $60 \%(1.60 \pm 0.09$ vs $1.00 \pm 0.06, p<0.01)$ in the 14 th day and by $116 \%(2.16 \pm 0.13$ vs $1.00 \pm 0.06, p<0.01)$ in the 28th day post MI.

Conclusions The expression level of miR-214 in myocardium and in plasma up-regulated in the progress of ventricular remodelling post $\mathrm{MI}$ in rat. The dynamic change of miR-214 may potentially become a new biomarker in ventricular remodelling post MI.

\section{e0052 VALSARTAN REVERSED VASCULAR FIBROSIS THROUGH THE BLOCKADE OF THE AT1-MEDIATED TGF- $\beta$ /SMAD SIGNAL PATHWAY IN THE FAT-FED, STREPTOZOTOCIN- TREATED RATS}

doi:10.1136/hrt.2010.208967.52

Sun Hui, Zhang Wei, Zhong Ming, Zhao Yong, Su Guohai, Zhang Yun. The Key Laboratory of Cardiovascular Remodelling And Function Research, Chinese Ministry of Education And Chinese Ministry of Public Health, Shandong University, Qilu Hospital, Ji'nan, China

Objective Angiotensin II (AII) and transforming growth factor- $\beta$ (TGF- $\beta$ ) are closely involved in the pathogenesis of diabetic complications. The aim of this study was to clarify the role of AII in the regulation of the TGF- $\beta$ system in diabetic vascular dysfunction. Methods Male Wistar rats were randomly divided into three groups: normal control, diabetic rats and valsartan group. Diabetes was induced by high-calorie diet for 4 weeks and a single intraperitoneal injection of streptozotocin (STZ) thereafter. The expression of TGF$\beta 1 /$ Smads signalling was analysed by real-time reverse transcriptasePCR and immunohistochemistry in aorta of three groups.

Results Compared with control group, the expression of both TGF- $\beta$ I $(27.4013 \pm 10.49256$ vs $15.1254 \pm 6.64343, p<0.01)$, TGF- $\beta$ receptor types II $(28.0173 \pm 10.22042$ vs $10.0561 \pm 8.22275, \mathrm{p}<0.01)$ and activation of the smad2/3 (31.4029 \pm 10.44721 vs $12.8769 \pm 6.98547$, $\mathrm{p}<0.001)$ signalling pathway were up-regulated in the vasculature in diabetic rats. Compared with diabetic group, active TGF- $\beta$ $(18.5682 \pm 10.29359$ vs $27.4013 \pm 10.49256, \mathrm{p}<0.05)$ and $S m a d 2 / 3$ $(20.5209 \pm 7.82756$ vs $31.4029 \pm 10.44721, \mathrm{p}<0.01)$ protein levels were reduced in the aorta after the treatment of valsartan.

Conclusions Our results suggest that AT1 receptor antagonist has reversed vascular fibrosis through the blockade of the AT1-mediated TGF- $\beta /$ Smad signal pathway in the diabetic rats with vascular dysfunction. These observations may del support additional, beneficial effects of angiotensin receptor antagonists observed during del diabetic vascular complications.

Note: $\mathrm{n}=$ ? to be sent back.

\section{e0053 ELECTROPORATION-MEDIATED ANGIOTENSIN II TYPE 2 RECEPTOR GENE TRANSFECTED INTO RAT CAROTID ARTERIES AND THE EFFECTS OF AT2R GENE TRANSFER ON NEOINTIMAL HYPERPLASIA IN RAT CAROTID ARTERIES AFTER BALLOON ANGIOPLASTY}

doi:10.1136/hrt.2010.208967.53

Defeng Liu, Jianping Liu.

Aim To study the effects of Electroporation on the angiotensin II type 2 receptor (AT2R) transfected into rat carotid arteries and study the effects of AT2R gene transfer on neointimal hyperplasia in rat carotid arteries after balloon angioplasty.

Methods Electroporation-mediated AT2R gene transfected into rat carotid arteries after the establishment of rat carotid balloon injury restenosis model. The arteries were harvested at 5 days, 14 days and 21 days after gene transfer. The expression of AT2R in arteries and morphology analysis were evaluated by fluorescence microscope, immunohistochemistry, HE staining and in situ hybridisation.

Results Electroporation-mediated AT2R gene delivered into injured rat carotid arteries significantly up-regulated the levels of AT2R 